\title{
Overview of ATLAS Heavy Flavor Measurements
}

Sally Seidel

University of New Mexico

Epiphany2018 Conference, Krakow, 9 January 2018 
I. Introduction to ATLAS

II. b-Hadron Pair Production Cross-section

III. Prompt $\mathrm{J} / \psi$ Pair Production Cross-section

IV. $\psi(2 S) \rightarrow J / \psi \pi^{+} \pi^{-}$and $X(3872) \rightarrow J / \psi \pi^{+} \pi^{-}$Production

V. Angular Analysis of $B_{d}^{0} \rightarrow K^{*} \mu^{+} \mu^{-}$Decays 


\section{Measurement of b-hadron Pair Production Cross-section*}

Message: This total cross section is measured: $\quad \sigma\left(B\left(\rightarrow J / \psi\left[\rightarrow \mu^{+} \mu^{-}\right]+X\right) B(\rightarrow \mu+X)\right)$

Using it, 8 differential cross sections are obtained:

$\frac{1}{\sigma} \frac{d \sigma}{d \Delta R(J / \psi \mu)}$ separation between the $\mathrm{J} / \psi$ and the third $\mu$ in the azimuth-rapidity plane

- $\frac{1}{\sigma} \frac{d \sigma}{d m(J / \psi \mu)}\left[\mathrm{GeV}^{-1}\right]$ mass of the $\mathrm{J} / \psi \mu$ system

- $\frac{1}{\sigma} \frac{d \sigma}{d \Delta \phi(J / \psi \mu)}\left[\operatorname{rad}^{-1}\right]$ azimuthal separation $\Delta \phi$ between the $\mathrm{J} / \psi$ and the third $\mu$

$\frac{1}{\sigma} \frac{d \sigma}{d p_{T}(J / \psi \mu)}$

transverse momentum $\mathrm{p}_{\mathrm{T}}$ of the 3-muon system

$\frac{1}{\sigma} \frac{d \sigma}{d \Delta y(J / \psi \mu)}$ rapidity separation $\Delta y$ between the $\mathrm{J} / \psi$ and the third $\mu$ the list continues..... 
- $\frac{1}{\sigma} \frac{d \sigma}{d y_{\text {boost }}(J / \psi \mu)}$

- $\frac{1}{\sigma} \frac{d \sigma}{d p_{T}^{\mu \mu \mu} / m^{\mu \mu \mu}}$

- $\frac{1}{\sigma} \frac{d \sigma}{d m^{\mu \mu \mu} / p_{T}^{\mu \mu \mu}}$ magnitude $\mathrm{y}_{\text {boost }}$ of the avg. rapidity of the $\mathrm{J} / \psi$ and the third $\mu$

ratio of the $\mathrm{p}_{\mathrm{T}}$ to the invariant mass of the 3 -muon system,

and its inverse

These differential cross sections are compared to predictions from several event generators. 


\section{Motivation:}

- Factorization of QCD calculations into parton distribution functions, hard matrix elements, and soft parton shower components allows the heavy (b) quark mass to be introduced at different stages.

- Several schemes are possible for inclusion of the heavy quark masses

- Previous analyses of heavy flavor production highlighted disagreements among theoretical predictions and between predictions and data. This analysis constrains the options.

- The region of small-angle $b \bar{b}$ production is especially sensitive to details of the calculations but has previously been only loosely constrained by data.

- Searches for Higgs produced in association with a vector boson (VH) and decaying to $b \bar{b}$ rely on the modeling of the background $b \bar{b}+V$ 
- Trigger: 2 oppositely charged muons with a common vertex, $\mathrm{p}_{\mathrm{T}}(\mu)>4 \mathrm{GeV},|\eta(\mu)|<2.4,2.5$ $<\mathrm{m}(\mu \mu)<4.3 \mathrm{GeV}$

- Integrated luminosity $=11.4 \mathrm{fb}^{-1}$

- Primary vertex: $\geq 2$ tracks, each with $\mathrm{p}_{\mathrm{T}}>400 \mathrm{MeV}$, with largest summed $\mathrm{p}_{\mathrm{T}}^{2}$.

- Form the muon candidates:

- use combined inner detector and muon spectrometer tracks

- $\mathrm{p}_{\mathrm{T}}(\mu)>6 \mathrm{GeV},|\eta(\mu)|<2.5$

- J/ $\psi$ candidates:

- opposite-sign muon pairs with $|\eta(\mu)|<2.3$ and directional correspondence with the trigger-level candidate

- $2.6<\mathrm{m}(\mu \mu)<3.5 \mathrm{GeV}$

- If multiple candidates per event, choose the one with mass closest to $J / \psi_{\mathrm{PDG}}$.

- Third muon: choose the highest- $\mathrm{p}_{\mathrm{T}}$ one not included in the $\mathrm{J} / \psi$ reconstruction.

- The $J / \psi$ and the third $\mu$ may come from feed-down or cascade.

- The data are first compared to these simulations:

- Inclusive b-hadron pairs from PYTHIA8.186 (2->2 matrix element with parton shower); CTEQ6L1 pdf, AU2 tune; b quarks are massless in the pdf but the mass is reinstated during the shower; pile-up included with PYTHIA8 + MSTW2008 pdf + A2 tune.

- $p p \rightarrow b \bar{b}$ simulated with HERWIG++, CTEQ6L1, UE-EE5 tune; b-quarks are massive in the matrix element and in the parton shower.

- 4-momenta of photons near muon $\left(\Delta \mathrm{R}_{\eta}(\mu, \gamma)<0.1\right)$ added to muon 


\section{Analysis details (2)}

Corrections:

- for trigger efficiency including vertex recon and spatial overlap of muons

- for muon reconstruction efficiency

- To collect the $J / \psi$ 's produced in decays of b-hadrons:

- Define $L_{x y}$ : transverse distance between primary vertex (PV) and dimuon vertex, signed positively for momentum pointing away from primary vertex.

- Define pseudo-proper decay time:

$$
\tau \equiv \frac{L_{x y} \cdot m\left(J / \psi_{P D G}\right)}{p_{T}\left(\mu^{+} \mu^{-}\right)}
$$

- $\mathrm{J} / \psi^{\prime}$ s from most $\mathrm{b}$ decays are non-prompt, so to optimize for signal events, require $\tau>0.25 \mathrm{~mm} / \mathrm{c}$.

- simultaneous maximum likelihood fit to the distributions of dimuon mass and $\tau$.

- Extract \# non-prompt J/ $\psi^{\prime}$ s. 
- To select the third muon, reject bkgs: prompt muons, muons from charged $\pi / \mathrm{K}$ decay, fake muons from decay in flight and hadron shower leakage, muons combined with continuum (false) $\mathrm{J} / \psi$, and muons in pile-up.

- Discriminate third-muon signal from bkg with a simultaneous fit on 2 observables:

- transverse impact parameter significance

$$
S_{d_{0}} \equiv d_{0} / \sigma_{d_{0}}
$$

( $d_{0}$ is distance of closest approach of the muon track to the PV in the r- $\phi$ projection, with sign given by the sign of the angular momentum of the track around the beam at point of closest approach)

- Output of a boosted decision tree using kinematic variables related to track deflection significance, momentum balance, and $|\eta|$.

- Subtract 3 remaining irreducible bkgs from fitted yields:

- $B_{c} \rightarrow J / \psi+\mu+X$ (very small, taken from simulation)

- Semileptonic decays of c-hadrons not resulting from b-hadron feed-down

- "Sail through" charged $\pi / \mathrm{K}$ : traverses the detector to the muon spectrometer without interacting or decaying (mimics a muon, taken from simulation) 


\section{Analysis details (4)}

\section{Corrections:}

- for the $\tau$ requirement: extrapolate to full range

- for detector resolution on momentum and $\eta$ of muons. Issue: migration between bins and in/out of fiducial volume.

Repeat for every kinematic bin for each differential cross section.

Systematic uncertainties:

- Muon efficiency corrections to data

- $\mathrm{J} / \psi$ model

- Background components in the fits

Statistical uncertainties:

- On the data statistics

- On the third-muon templates taken from simulation

Luminosity uncertainty: $1.9 \%$ 
Result 1: $\sigma\left(B\left(\rightarrow J / \psi\left[\rightarrow \mu^{+} \mu^{-}\right]+X\right) B(\rightarrow \mu+X)\right)=17.7 \pm 0.1$ (stat) \pm 2.0 (syst) nb.

Result 2: Is the scale of $\alpha_{\mathrm{s}}$ during splitting set by relative $p_{T}$ or by mass?

Compare differential cross sections using 6 options in PYTHIA8 for the $g \rightarrow b \bar{b}$ splitting kernel (dominates small angle b-hadron production).

PYTHIA8 does not reproduce the shape of the angular distributions for any of the 6 options.

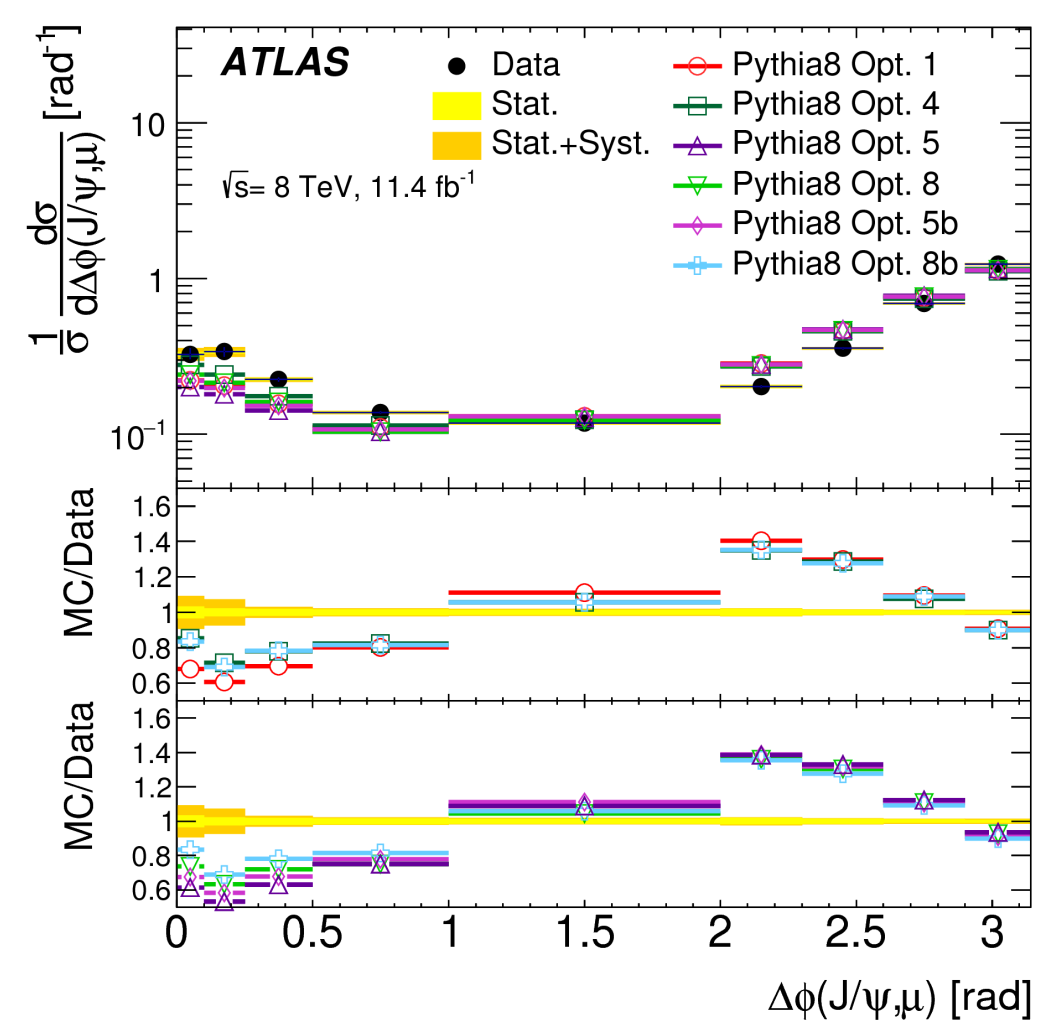

Some of the options of splitting function form and $\alpha_{\mathrm{s}}$ scale fit better to the mass or $\Delta \mathrm{R}$ distributions. Example:

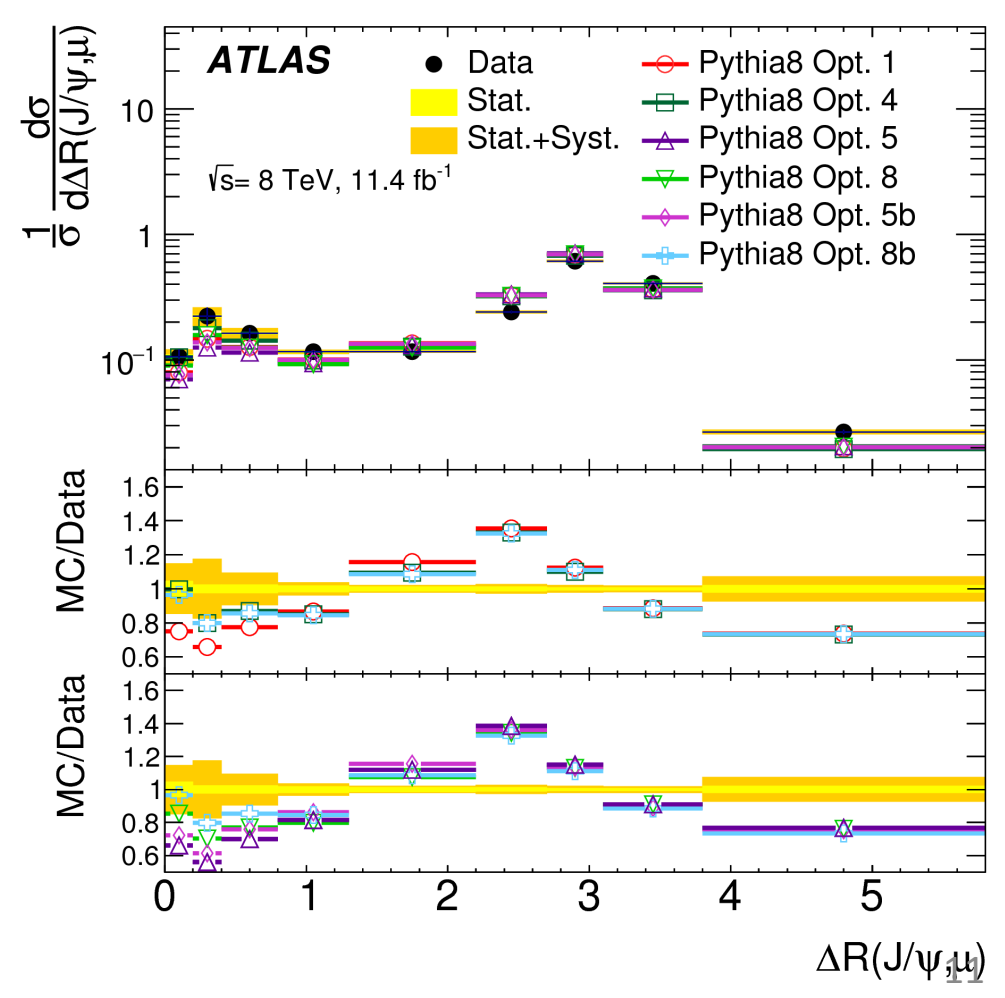




\section{Result 3:}

Extend the comparison of data to HERWIG++, SHERPA, and

MADGraph5_AMC@NLOv2.2.2+ PYTHIA8.186 parton shower model.

These cover a range of matrix elt calculations and parton shower models.

Consider options with 4 or 5 massless flavors. Compare all of these to PYTHIA8.

- HERWIG++ reproduces the $\Delta \mathrm{R}$ and $\Delta \phi$ graphs best.

- 4-massless flavors models $\Delta \mathrm{R}$ and $\Delta \phi$ better than 5 .

- $\Delta y$ spectrum is well modeled by MadGraph and SHERPA

- All models reproduce $y_{\text {boost }}$ well.

- 5-massless flavor MadGraph models low mass distribution better than 4 ,

- but 4-massless flavor MadGraph models high $\mathrm{p}_{\mathrm{T}} / \mathrm{m}$ best.

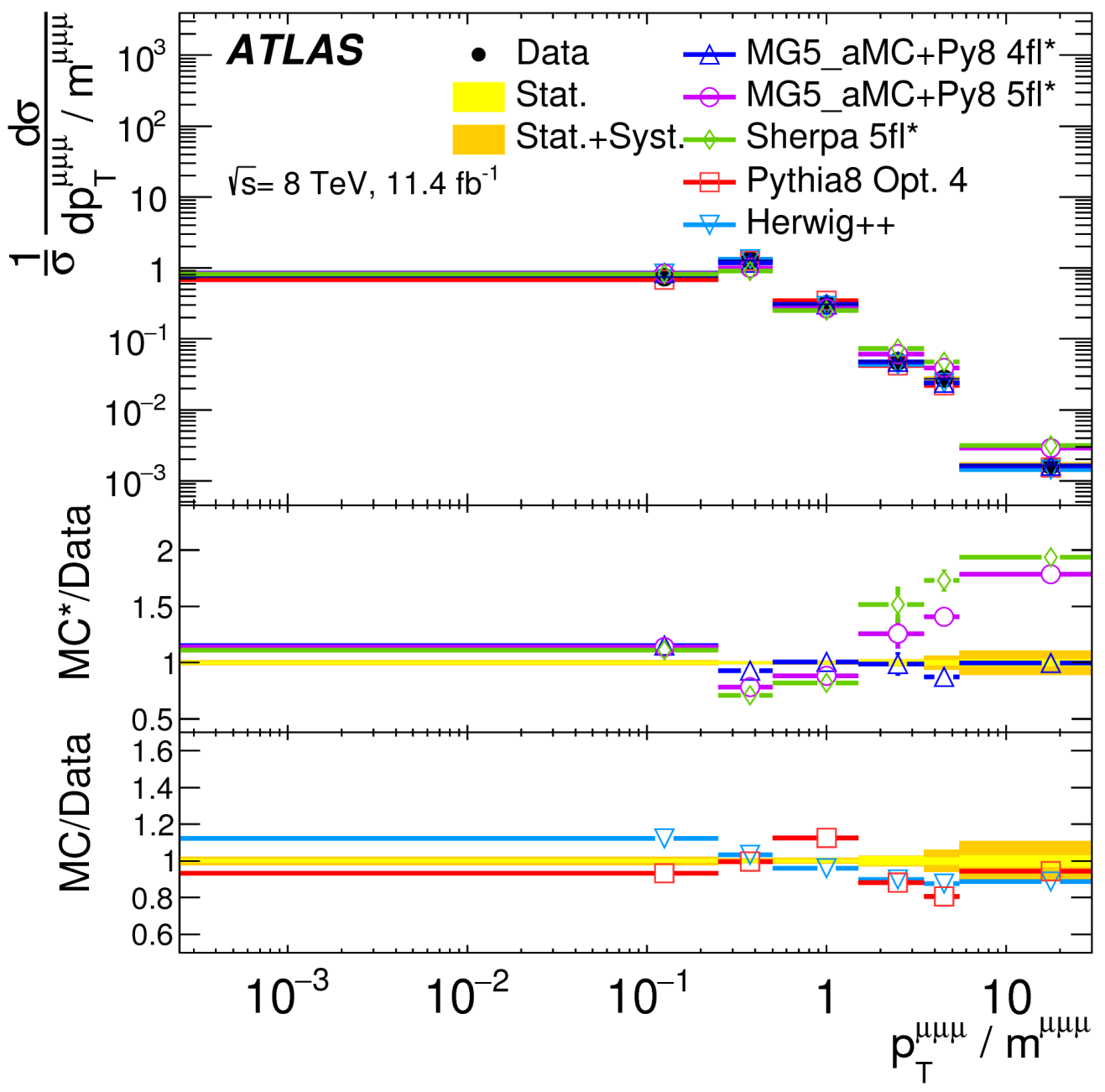




\section{Conclusions:}

- Considering all distributions, the 4-massless flavor prediction from MadGraph5_AMC@NLO+PYTHIA8 best describes the data.

- Predictions of PYTHIA8 and HERWIG++ are comparable.

- Among PYTHIA8 options studied, the $\mathrm{p}_{\mathrm{T}}$-based splitting kernel is best. 


\section{Measurement of Prompt J/ $\psi$ Pair Production Cross-section*}

Message: The cross section for production of 2 prompt centrally-produced $\mathrm{J} / \psi$ mesons is measured. "Prompt" means: produced at a point consistent with the primary vertex, not as a product of the decay of a long-lived hadron.

Differential cross sections are produced as a function of:

- $\mathrm{p}_{\mathrm{T}}$ of the lower- $\mathrm{p}_{\mathrm{T}}$ meson (called " $\mathrm{J} / \psi_{2}$ ") - Measurements use subleading meson $\mathrm{J} / \psi_{2}$ to access full kinematic region.

- $\mathrm{di}-\mathrm{J} / \psi \mathrm{p}_{\mathrm{T}}$

- $\mathrm{di}-\mathrm{J} / \psi$ mass

- $\Delta y$ between the 2 mesons

- $\Delta \phi$ between the 2 mesons

Characterization of kinematic correlations between the $2 \mathrm{~J} / \psi$ 's is used to extract the fraction of prompt pair events arising from double parton scattering.

Total and double parton scattering cross sections are compared with predictions.

The effective cross section of a double parton scattering is measured.

This is the first such measurement at $8 \mathrm{TeV}$, and it probes a different kinematical range from previous $(1.96 \mathrm{TeV}$ and $7 \mathrm{TeV})$ measurements. *Eur. Phys. J. C (2017) 77:76. 
What the events look like: each shaded circle is a $\mathrm{J} / \psi$ meson. Di-J/ $\psi$ 's can be produced from single parton ( $g$-g) scattering ("SPS") or from double parton scattering ("DPS").

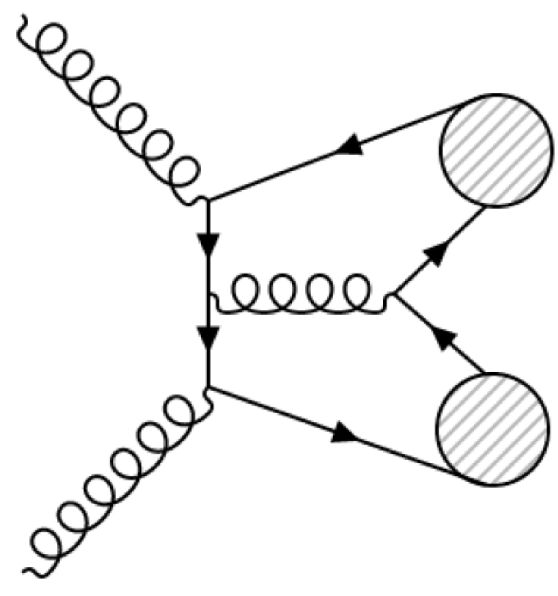

LO SPS

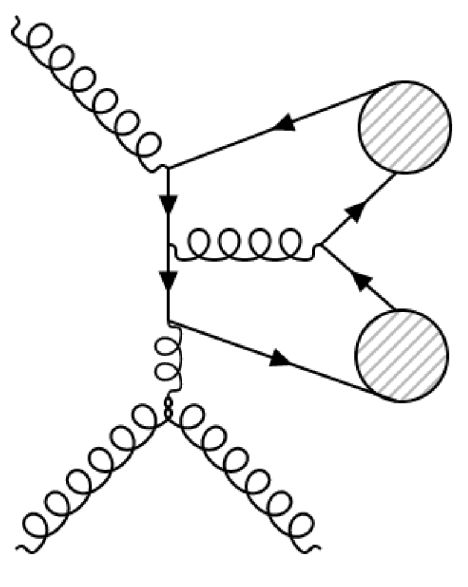

NLO SPS, color singlet $\mathrm{J} / \psi$



LO SPS, color octet cं

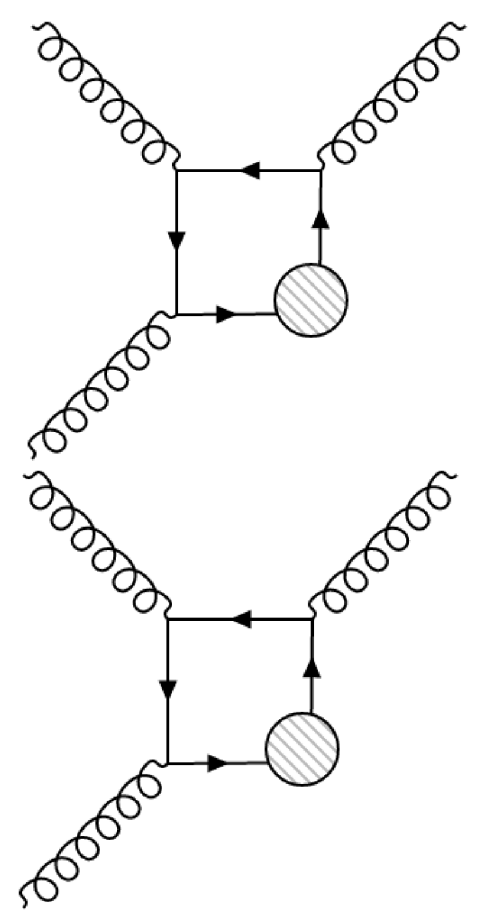

DPS 


\section{Motivation:}

Goal \#1: measure the fraction of events that result from double parton scattering. The DPS cross section is sensitive to the spatial distribution of gluons in the proton.

Goal \#2: use the fraction of DPS events $f_{D P S}$ to measure the effective cross section of DPS. Effective cross section is:

$$
\sigma_{e f f}=\frac{1}{2} \frac{\sigma_{J / \psi}^{2}}{f_{D P S} \cdot \sigma_{J / \psi J / \psi}}
$$

It relates the production cross section of the 2 individual interactions to the total production cross section. Testing correlations of non-perturbative origin between the partons in a DPS may improve understanding of non-perturbative QCD.

Goal \#3: DPS can be modeled and subtracted to provide input to SPS quarkonium production models. Quarkonium production is a background to new physics searches. Make comparisons between the data and various production models using different techniques to compute di-J/ $\psi$ production at LO, NLO, NLO color singlet NRQCD without loops (NLO*), and intrinsic parton transverse momentum fractions. 


\section{Details of the analysis (1)}

- Integrated luminosity $=11.4 \mathrm{fb}^{-1}$

- Accept prompt-prompt mesons produced directly or through feed-down from $\psi(2 \mathrm{~S})$ decay

- Dimuon trigger, each muon's $\mathrm{p}_{\mathrm{T}}>4 \mathrm{GeV} ; 2.5<\mathrm{m}\left(\mu^{+} \mu^{-}\right)<4.3 \mathrm{GeV}$

- Reconstruction:

- $\geq 3$ muons in the muon spectrometer data

- Record $\left|\mathrm{d}_{\mathrm{z}}\right|$ of $2 \mathrm{~J} / \psi$ decay vertices projected onto the beam axis

- $\left|\eta^{\mu}\right|<2.3, \mathrm{p}_{\mathrm{T}}{ }^{\mu}>2.5 \mathrm{GeV}$

- $2.8<\mathrm{m}(\mu \mu)<3.4 \mathrm{GeV}$

- $\left|\mathrm{y}^{\mathrm{J} / \psi}\right|<2.1, \mathrm{p}_{\mathrm{T}}^{\mathrm{J} / \psi}>8.5 \mathrm{GeV}$

- For each $\mathrm{J} / \psi$ candidate, find the signed transverse decay length $\mathrm{L}_{\mathrm{xy}}$ (recall from page 8)

- Because $\mathrm{J} / \psi$ mass resolution is worse in forward region, measure cross section separately for 2 rapidity regions: $\left|\mathrm{y}^{\mathrm{J} / \psi}\right|<1.05$ ("central") and $1.05<\left|\mathrm{y}^{\mathrm{J} / \psi}\right|<2.1$ ("forward") 


\section{Analysis details (2)}

\section{Signal extraction procedure:}

- Weight each event by efficiency of trigger, reconstruction, and selection, and by geometrical acceptance.

- First find all di-J/ $\psi$ events:

- Build a 2-d distribution of the $\mathrm{m}\left(\mathrm{J} / \psi_{1}\right)$ vs. $\mathrm{m}\left(\mathrm{J} / \psi_{2}\right)$ from inclusive single $\mathrm{J} / \psi$ events. Signal for each $\mathrm{J} / \psi$ is modeled by Crystal Ball function, bkg (muons from semileptonic decays of $b$-hadrons and from continuum) is modeled by polynomial

- Fit the data to this $2-\mathrm{d}$ probability density function. Subtract this non-J/ $\psi \mathrm{bkg}$. What remains is inclusive di-J/ $\psi$ signal (prompt and non-prompt).

- To extract only prompt-prompt ("PP") events from that inclusive di-J/ $\psi$ sample:

- Construct $2 \mathrm{~L}_{\mathrm{xy}}$ probability distributions from the inclusive $\mathrm{J} / \psi$ sample - one for prompt-prompt and one for nonprompt-nonprompt. Prompt events have $\mathrm{L}_{\mathrm{xy}}$ consistent with resolution, non-prompt with an exponential (decay constant $\tau$ ). Mixed prompt-nonprompt events are negligible.

- Classify events according to the rapidity bins (central or forward) of the $2 \mathrm{~J} / \psi$ 's and apply bin-specific decay constants $\tau$ to the exponentials.

- For each event, plot $\mathrm{L}_{\mathrm{xy}}$ of $\mathrm{J} / \psi_{1}$ versus $\mathrm{L}_{\mathrm{xy}}$ of $\mathrm{J} / \psi_{2}$ and compare it to the PP and NPNP PDFs, then classify it.

- Divide the PP-weighted PDF by the full PDF to get the likelihood that the event is PP as a function of its mesons' values for $\mathrm{L}_{\mathrm{xy}}$ and rapidity.

- Subtract pileup bkg: remove events with $\left|\mathrm{d}_{\mathrm{z}}\right|>1.2 \mathrm{~mm}$. 
- Determine DPS fraction:

- Construct a data-driven DPS template by combining $\mathrm{J} / \psi$ mesons from different random events in the di-J/ $\psi$ sample.

- Construct a data-driven SPS template by subtracting the DPS template from the di$\mathrm{J} / \psi$ samples' $\Delta \mathrm{y}$ vs. $\Delta \phi$ distribution.

- Normalize the DPS sample to the data in the region $\Delta y>1.8$ and $\Delta \phi<\pi / 2$ (where

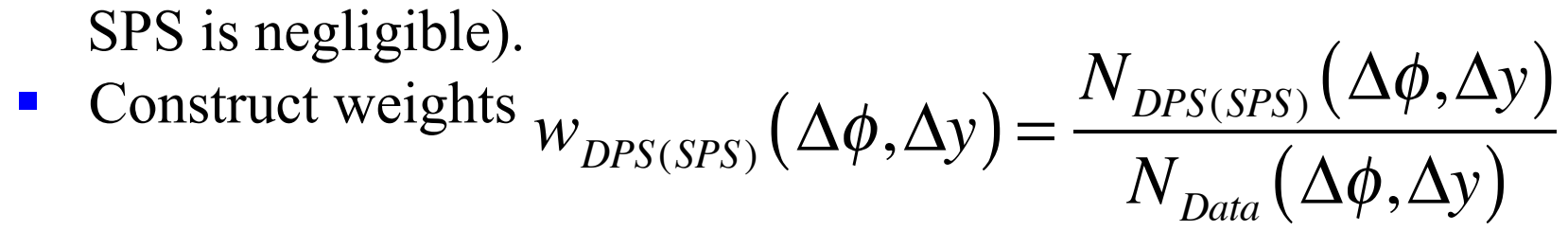

- For every event, apply these weights, apply the PP weight, fit to template of $\mathrm{m}\left(\mathrm{J} / \psi_{1}\right)$ vs. $\mathrm{m}\left(\mathrm{J} / \psi_{2}\right)$ in bins of the chosen variable, extract PP SPS signal and PP DPS signals, compute $\mathrm{f}_{\mathrm{DPS}}$.

- For this fixed $\mathrm{f}_{\text {DPS }}$, compare distribution to LO DPS and NLO* SPS model distributions.

- Extract effective cross section 


\section{Analysis details (4)}

\section{Corrections:}

- Dimuon trigger efficiency including (1) correlations between vtx resolution and oppositesign requirement and (2) muons overlapped and unresolvable by the trigger

- Muon recon efficiency

- Kinematic acceptance (from simulation - effect of $\mathrm{p}_{\mathrm{T}}$ and $\eta$ cuts on fiducial region)

- Signal efficiency on $\mathrm{d}_{\mathrm{z}}$ and $\mathrm{L}_{\mathrm{xy}}$.

- $\mathrm{p}_{\mathrm{T}}$-dependence of reconstructed mass and mass resolution of $\mathrm{J} / \psi$

\section{Systematic uncertainties:}

Trigger selection, muon recon, kinematic acceptance, mass model developed from inclusive $\mathrm{J} / \psi$ sample, $\mathrm{J} / \psi$ mass and width bias function of $\mathrm{p}_{\mathrm{T}}$, prompt-prompt model (from inclusive $\mathrm{J} / \psi$ sample) dependence on $\mathrm{p}_{\mathrm{T}}$, pile-up, $\mathrm{J} / \psi$ to dimuon branching fraction, luminosity, DPS model and binning. 


\section{Results:}

- Prompt-prompt cross sections measured:

$$
\begin{aligned}
& \sigma_{\text {central }}(J / \psi J / \psi)=82.2 \pm 8.3 \text { (stat.) } \pm 6.3 \text { (syst.) } \pm 0.9 \text { (BF) } \pm 1.6 \text { (lumi) pb } \\
& \sigma_{\text {forward }}(J / \psi J / \psi)=78.3 \pm 9.2 \text { (stat.) } \pm 6.6 \text { (syst.) } \pm 0.9 \text { (BF) } \pm 1.5 \text { (lumi) pb }
\end{aligned}
$$

- Data are compared to theoretical distributions. ${ }^{\S}$ Shapes of DPS distributions are consistent with models. For SPS, the data distributions in $\Delta \mathrm{y},|\Delta \phi|, \mathrm{m}(\mathrm{J} / \psi \mathrm{J} / \psi)$, and $\mathrm{p}_{\mathrm{T}}(\mathrm{J} / \psi \mathrm{J} / \psi)$ are wider than predicted by the NLO calculation.

- Data and predictions especially diverge for $\Delta y>1.8$. This may indicate a large effect due to $\mathrm{k}_{\mathrm{T}}$ or contributions via feeddown from color-singlet $\psi(2 \mathrm{~S})$.

${ }^{\S}$ LO DPS: C. Borschensky and A. Kulesza, arXiv: 1610.00666 [hep-ph]; NLO* SPS: J.P. Lansbert, H.S. Shao, Phys. Lett. B 751, 479 (2015) and PRL 111, 122001 (2013).

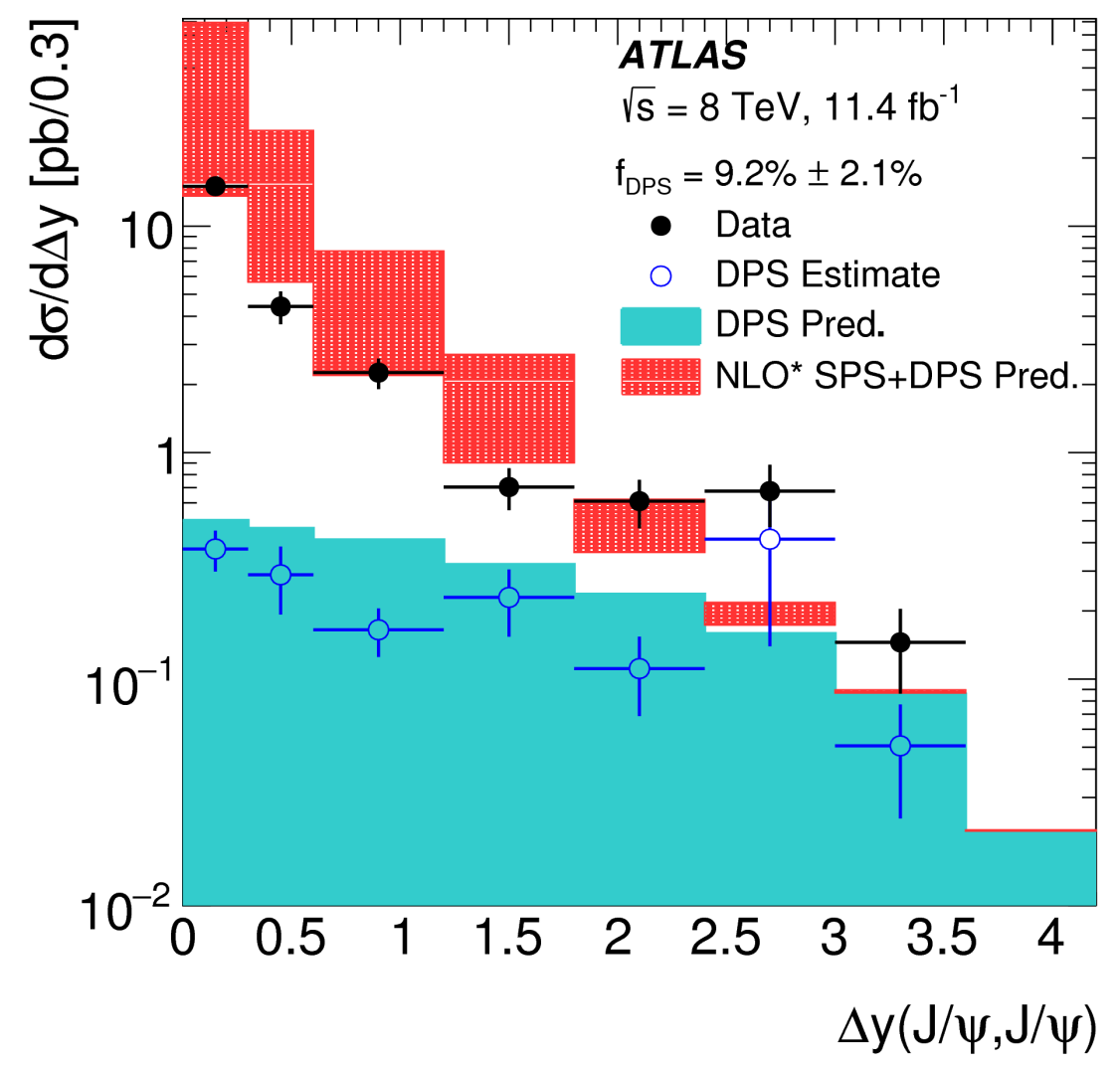



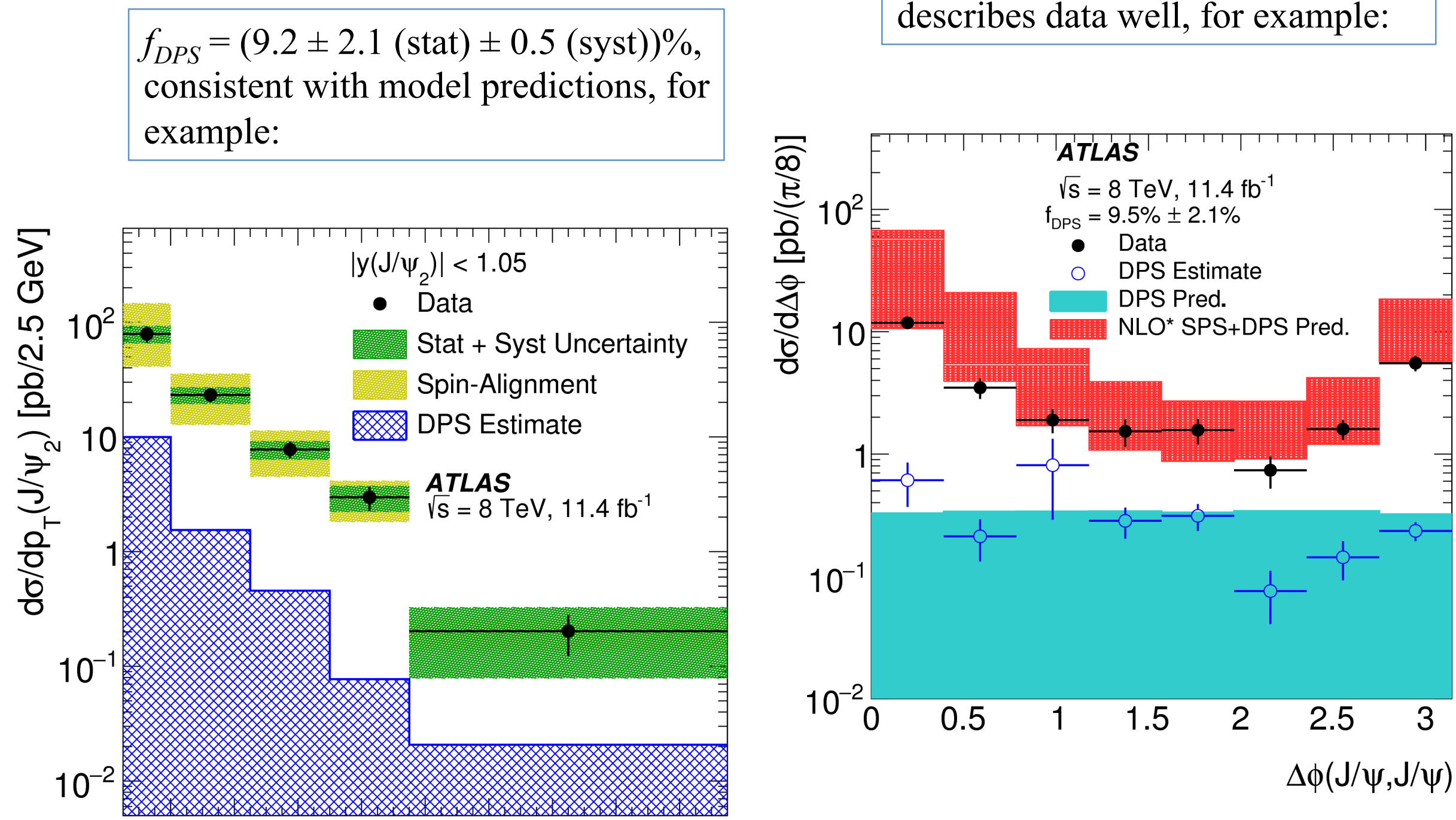

$\mathrm{p}_{\mathrm{T}}\left(\mathrm{J} / \psi_{2}\right)[\mathrm{GeV}]$

NLO* with LO DPS+NLO color singlet SPS w/o loops generally describes data well, for example:

\section{ATLAS}

$\sqrt{\mathrm{s}}=8 \mathrm{TeV}, 11.4 \mathrm{fb}^{-1}$$$
P_{T}{ }_{2}
$$

$\begin{array}{lllllllll}10 & 12 & 14 & 16 & 18 & 20 & 22 & 24 & 26\end{array}$ 


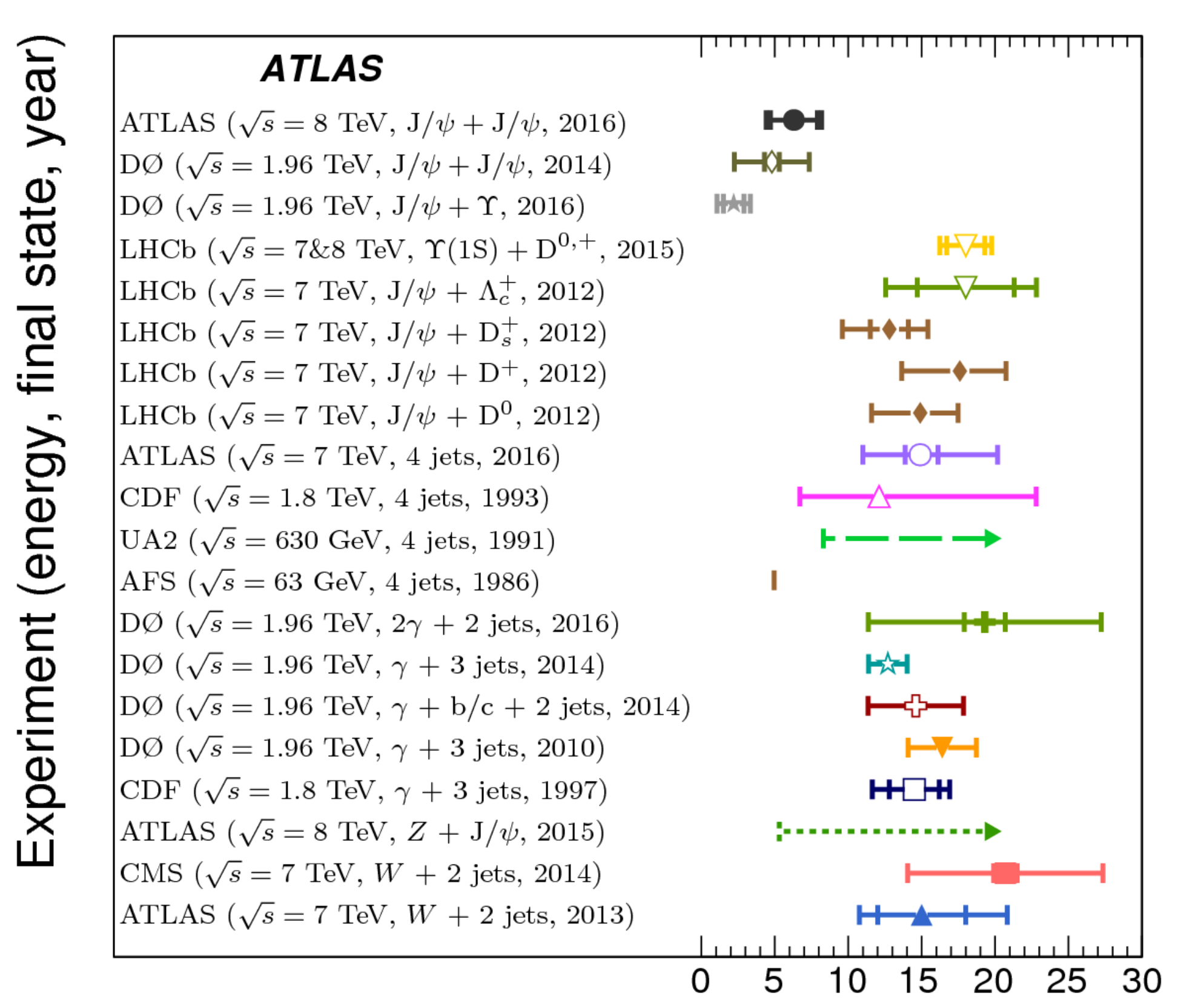

$\sigma_{e f f}=(6.3 \pm 1.6$ (stat) \pm 1.0 (syst) $\pm 0.1(\mathrm{BF}) \pm 0.1$ (lumi) $) \mathrm{mb}$ 


\section{Measurements of $\psi(2 S) \rightarrow J / \psi \pi^{+} \pi^{-}$and $X(3872) \rightarrow J / \psi \pi^{+} \pi^{-}$ Production*}

\section{Message:}

Differential cross sections of $\mathrm{X}(3872)$ and $\psi(2 \mathrm{~S})$ are measured and compared to models, for prompt and non-prompt production.

The ratio of production cross sections $\mathrm{X}(3872) / \psi(2 \mathrm{~S})$ is measured.

The fraction of non-prompt X(3872) and the fraction of non-prompt $\psi(2 S)$ are measured.

The non-prompt $X(3872)$ sample requires 2 lifetimes in the fit. The short lifetime component involves $\mathrm{X}(3872)$ 's produced in $\mathrm{B}_{\mathrm{c}}$ decays.

The invariant mass of the dipion system in the $J / \psi \pi^{+} \pi^{-}$final state is measured and found to be consistent with the process $\rho^{0} \rightarrow \pi^{+} \pi^{-}$. 


\section{Motivation:}

This study examines:

- the production mechanisms for these hidden charm states: direct versus feed-down from one or more heavy hadrons.

- the production mechanism of the dipion in the final state

The current best model for the $\mathrm{X}(3872)$ is a mixed $\chi_{c 1}(2 P)-D^{0} \bar{D}^{* 0}$ state. 


\section{Details of the analysis (1)}

- Integrated luminosity $=11.4 \mathrm{fb}^{-1}$

- Trigger: dimuons fitted to a common vertex

- Reconstruction:

- Muons well matched to trigger objects

- $\mathrm{p}_{\mathrm{T}}(\mu)>4 \mathrm{GeV},|\eta(\mu)|<2.3, \mathrm{~m}(\mu \mu)$ within $\mathrm{m}_{\mathrm{J} / \psi} \pm 120 \mathrm{MeV}$

- Find $\mathrm{J} / \psi \pi^{+} \pi^{-}$candidates:

- Constrain $\mathrm{m}(\mu \mu)$ to $\mathrm{m}(\mathrm{J} / \psi)_{\mathrm{PDG}}$, then assign pion masses to 2 additional oppositely charged non-muon tracks and fitted to a common vertex with the muons. $\mathrm{p}_{\mathrm{T}}(\pi)>0.6 \mathrm{GeV},|\eta(\pi)|$ $<2.4$.

- $\mathrm{J} / \psi \pi^{+} \pi^{-}$rapidity $|\mathrm{y}|<0.75$; $10<\mathrm{p}_{\mathrm{T}}<70 \mathrm{GeV}$.

- $\Delta \mathrm{R}\left(\mathrm{J} / \psi, \pi^{ \pm}\right)<5$ : the angular distance between momenta of the dimuon system and each candidate.

- Require: $\mathrm{Q}=\mathrm{m}\left(\mathrm{J} / \psi \pi^{+} \pi^{-}\right)$- $\mathrm{m}(\mathrm{J} / \psi)$ $\mathrm{m}(\pi \pi)<0.3 \mathrm{GeV}$ : suppresses combinatorial bkg while saving $90 \%$ of signal.

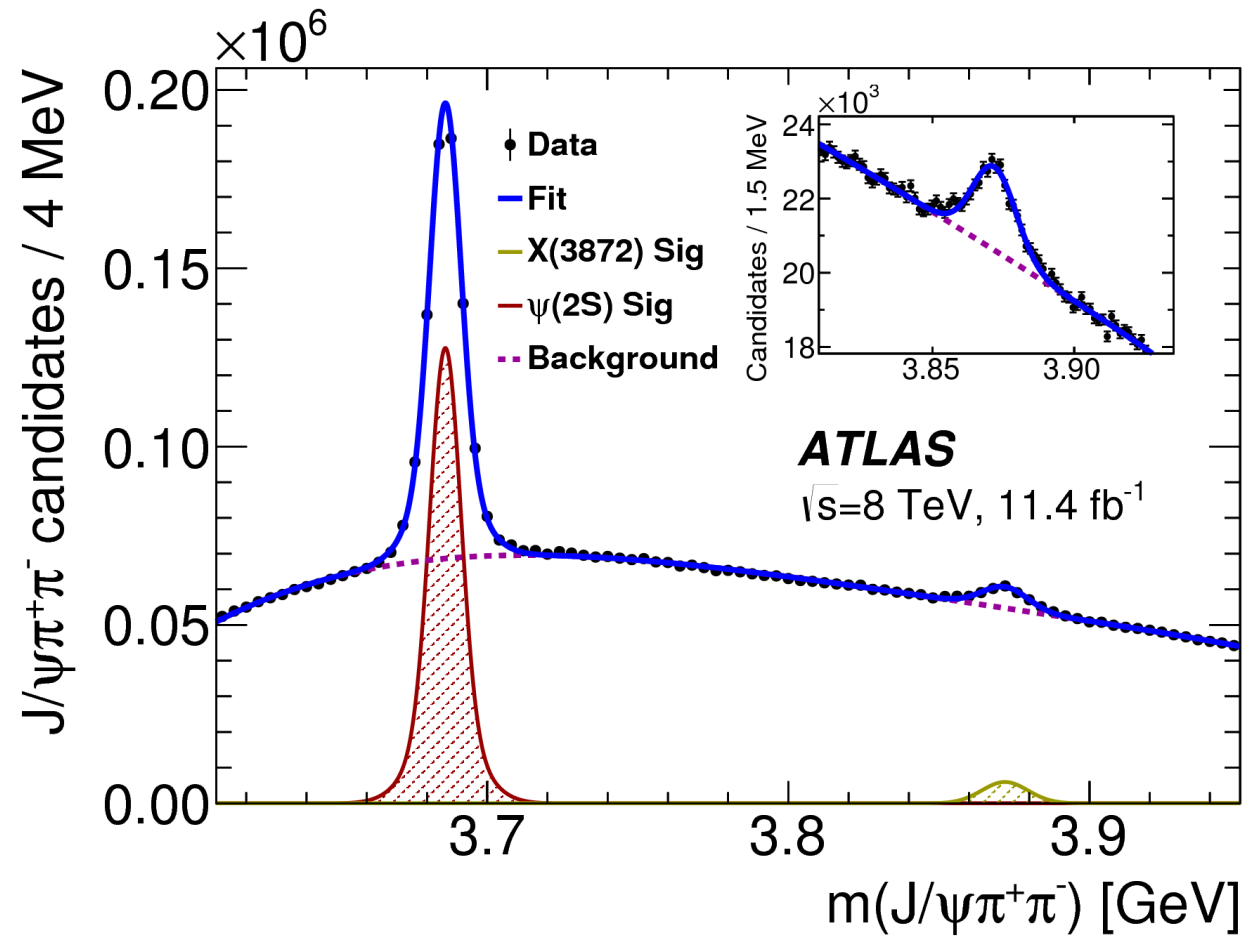




\section{Analysis details (2)}

- Bin candidates in $\mathrm{p}_{\mathrm{T}}$.

- Weight each candidate for $\mathrm{p}_{\mathrm{T}^{-}}$and $\eta$-dependent selection and recon efficiencies

- Subdivide candidates in each $\mathrm{p}_{\mathrm{T}}$ bin according to pseudo-proper lifetime where

$$
\begin{gathered}
\tau=\frac{L_{x y} m}{c p_{T}} \\
L_{x y}=\frac{\vec{L} \cdot \vec{p}_{T}}{p_{T}}
\end{gathered}
$$

$\vec{L}$ is the vector pointing from the PV to the $\mathrm{J} / \psi \pi^{+} \pi^{-}$vertex.

- Define 4 lifetime intervals.

- Fit data distribution in each lifetime interval to a function with 2 double-Gaussian signal functions (the $\psi(2 \mathrm{~S})$ and the $\mathrm{X}(3872)$, polynomial bkg. Extract signal yields $\mathrm{Y}$. 


\section{Analysis details (3)}

- Apply yields Y to find double differential cross sections $\times$ branching ratios, for $i=$ $\mathrm{X}(3872)$ or $\psi(2 \mathrm{~S})$ :

$$
B\left(i \rightarrow J / \psi \pi^{+} \pi^{-}\right) B\left(J / \psi \rightarrow \mu^{+} \mu^{-}\right) \cdot \frac{d^{2} \sigma(i)}{d p_{T} d y}=\frac{Y(i)}{\Delta p_{T} \Delta y \int L d t}
$$

- First fit data in each $\mathrm{p}_{\mathrm{T}}$ bin assuming one prompt component and one non-prompt $\left(\tau_{\text {eff }}\right)$ component. Observe: $\tau_{\text {eff }}$ is different for low- $p_{T} X(3872)$ decays. Do these proceed by a different mechanism?

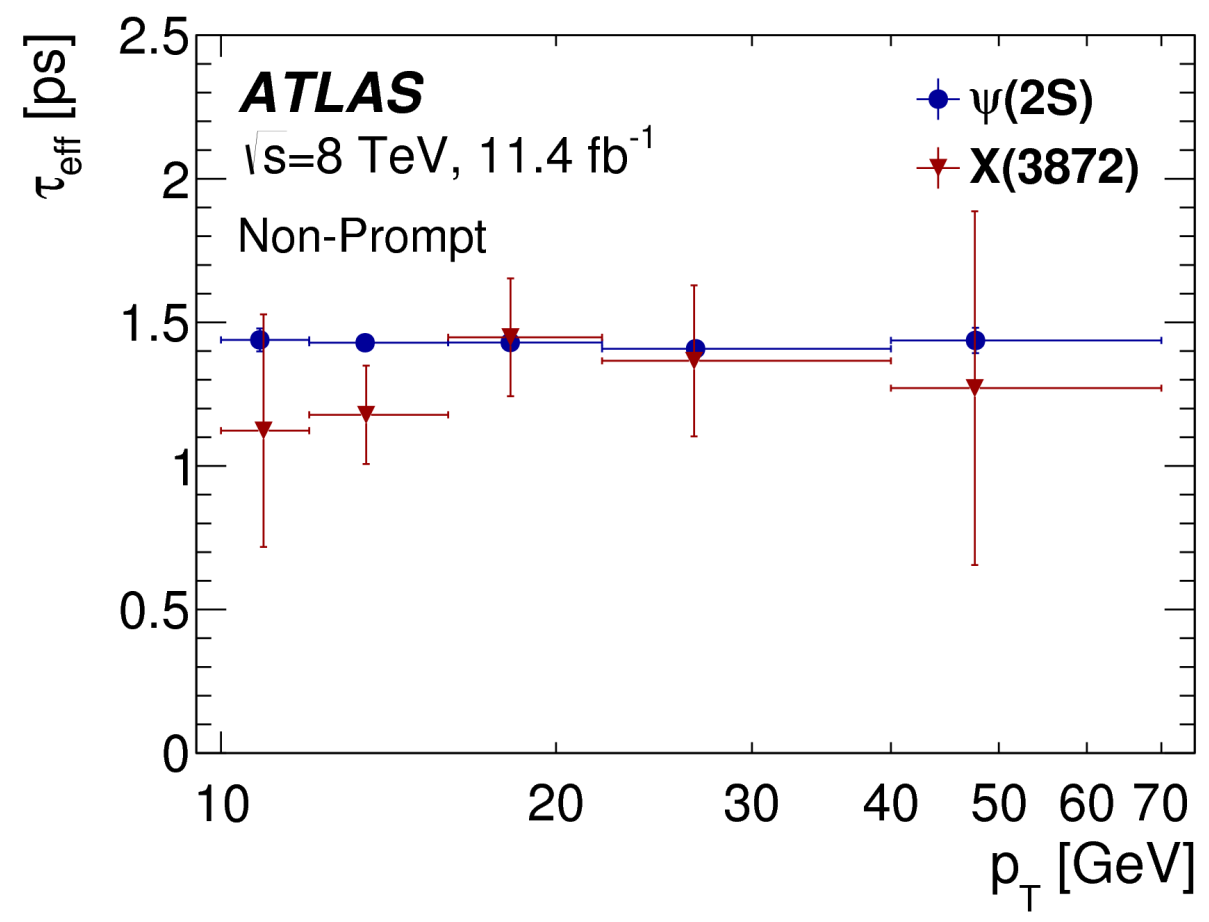




\section{Analysis details (4) and results:}

- Try 2 lifetimes for the non-prompt decays. (Short component: from $\mathrm{B}_{\mathrm{c}}$ decays, long component from all other $\mathrm{B}^{ \pm}, \mathrm{B}^{0}, \mathrm{~B}_{\mathrm{s}}$, and b-baryons.)

- Observe: no short-lived non-prompt component in $\psi(2 \mathrm{~S})$ production.

- For X(3872), short-lived non-prompt fraction is 25\%:

$$
\frac{\sigma\left(p p \rightarrow B_{c}\right) \cdot B\left(B_{c} \rightarrow X(3872)\right)}{\sigma(p p \rightarrow \text { non-prompt } X(3872))}=(25 \pm 13(\text { stat }) \pm 2(\text { syst }) \pm 5(\text { spin })) \%
$$

- Measure ratio:

$$
\left.R=\frac{B(B \rightarrow X(3872)+\text { any }) \cdot B\left(X(3872) \rightarrow J / \psi \pi^{+} \pi^{-}\right)}{B(B \rightarrow \psi(2 S)+\text { any }) \cdot B\left(\psi(2 S) \rightarrow J / \psi \pi^{+} \pi^{-}\right)}=(3.57 \pm 0.33 \pm 0.11)\right) \times 10^{-2}
$$

This ratio is below the value inferred from the ratio of Tevatron data (numerator) to the world average of branching fractions (denominator): $0.18 \pm 0.08$. 


\section{Results on differential} cross sections:
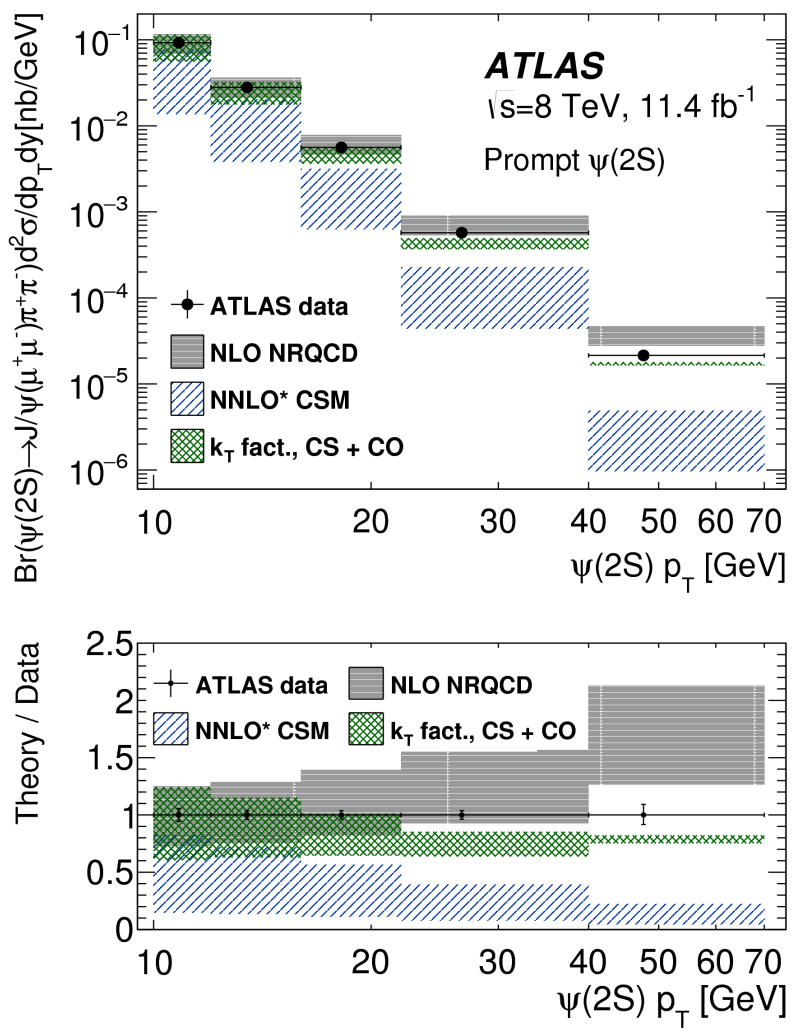

For $\psi(2 S)$ prompt and nonprompt:

- Generally good agreement between data and NLO NRQCD using long distance matrix elements derived from Tevatron data, below highest $\mathrm{p}_{\mathrm{T}}$

- $\mathrm{k}_{\mathrm{T}}$ factorization model including color-octet contributions tuned on 7 TeV CMS data + color singlet contributions describes data well but underestimates at highest $\mathrm{p}_{\mathrm{T}}$.

- NNLO* color singlet model agrees at low $\mathrm{p}_{\mathrm{T}}$.
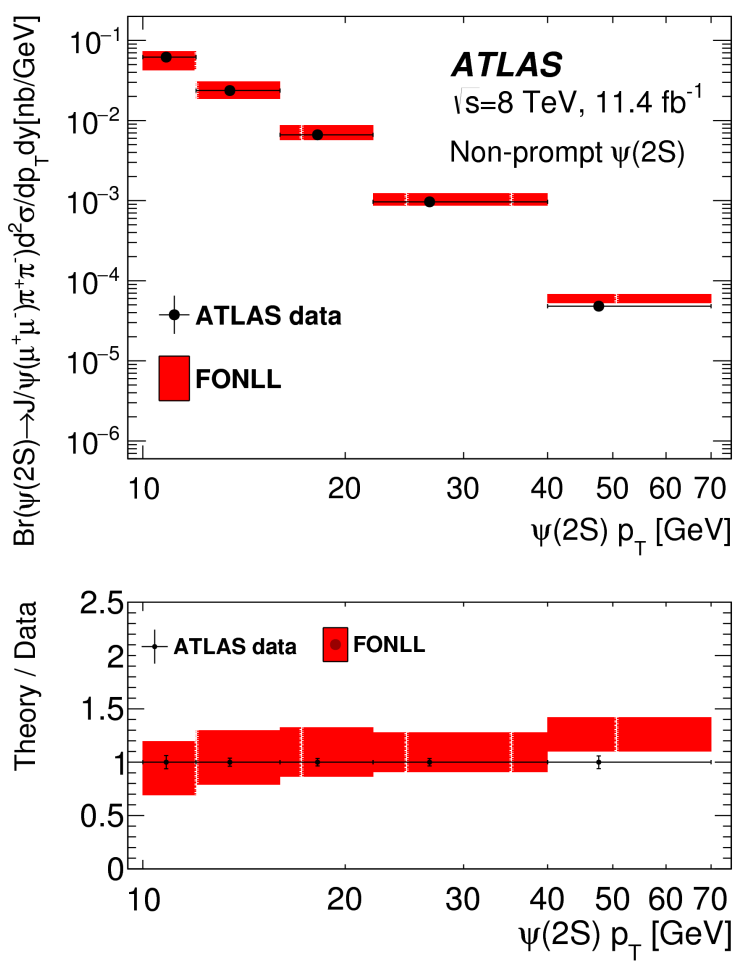

For non-prompt $\psi(2 S)$ : good agreement with FONLL over full $\mathrm{p}_{\mathrm{T}}$ range 
Results on differential cross sections, continued:

For prompt $X(3872)$ : described adequately by NRQCD as $\chi_{c 1}(2 P)-D^{0} \bar{D}^{* 0}$ mixture
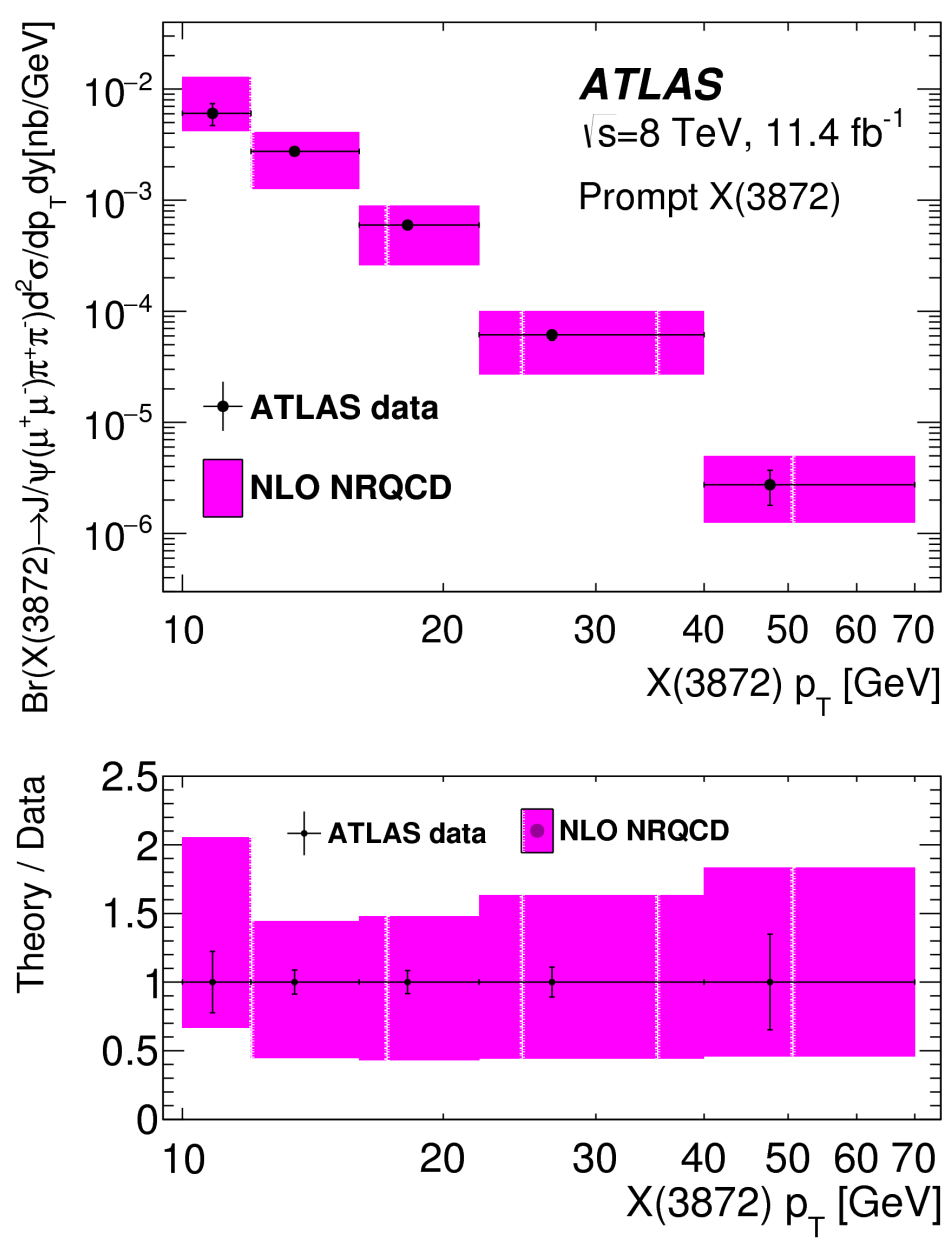

For non-prompt X(3872): FONLL model overestimates data by factor $4-8$, increasing with $\mathrm{p}_{\mathrm{T}}$
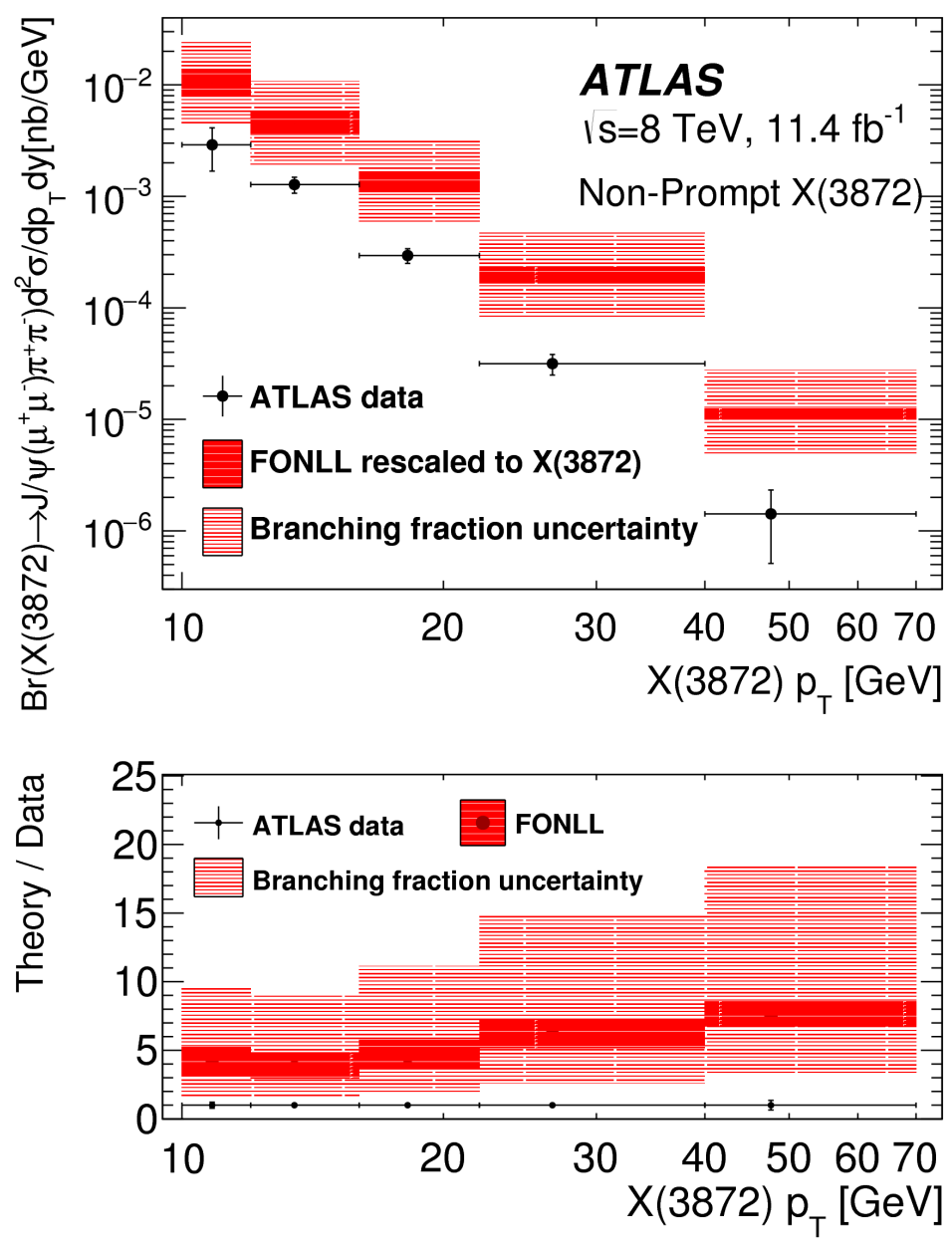
Results on differential cross sections, continued:

Using the normalized differential decay width in bins of dipion invariant mass, we see that phase space decay is disfavored: the pion production occurs through $\rho^{0} \rightarrow \pi^{+} \pi^{-}$.
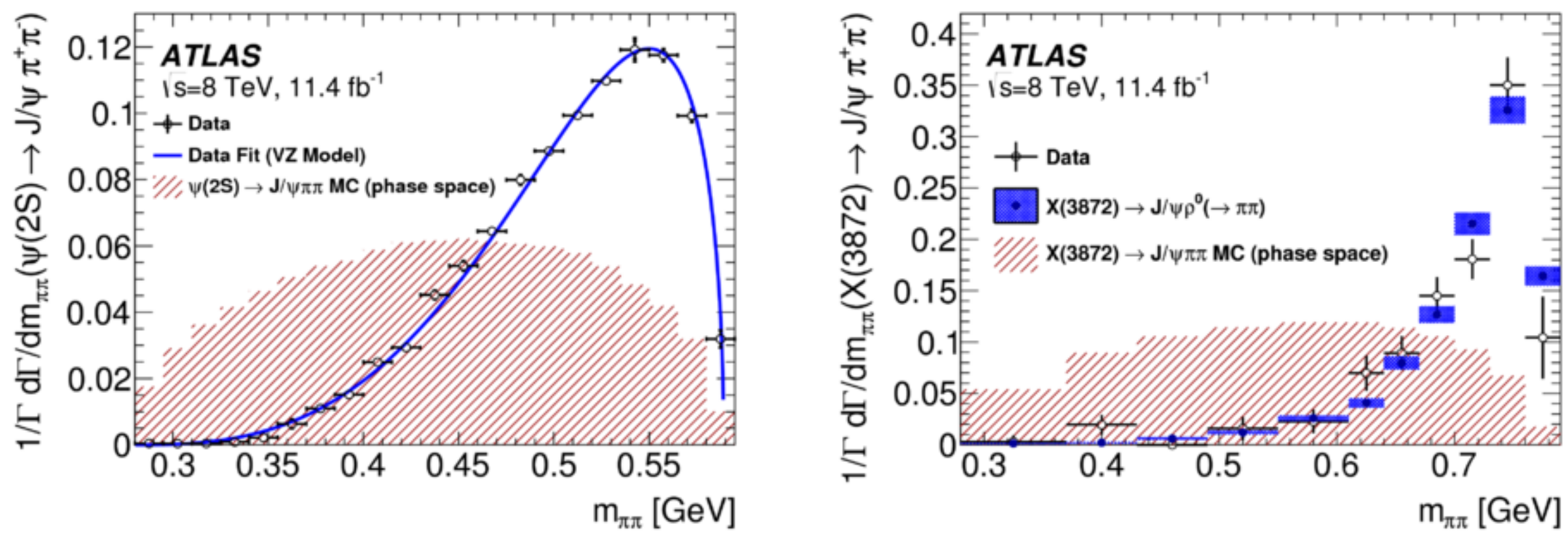


\section{Angular Analysis of $B_{d}^{0} \rightarrow K^{*}\left(\rightarrow K^{+} \pi^{-}\right) \mu^{+} \mu^{-}$Decays*}

\section{The message:}

The longitudinal polarization of the $K^{*}$ is measured and compared to theoretical predictions.

This polarization can be influenced by penguin diagrams involving new physics.

Hadron form factors dominate the prediction at leading order.

LHCb has adopted a method $\$$ for minimizing uncertainties in hadron form factors in this measurement. LHCb observes a 3.4 sigma deviation from Standard Model calculations. The $\mathrm{LHCb}$ method is used here.

*ATLAS-CONF-2017-023 (3 April 2017).

$\S$ LHCb Collaboration, PRL 111 (2013) 191801.

^LHCb Collaboration, JHEP 02 (2016) 104. 


\section{The method:}

- 3 angular variables:

- $\theta_{\mathrm{K}}$, between the $\mathrm{K}^{+}$and the direction opposite the $\mathrm{B}_{\mathrm{d}}$, in the $\mathrm{K}^{*}$ frame

- $\theta_{\mathrm{L}}$, between the $\mu^{+}$and the direction opposite the $\mathrm{B}_{\mathrm{d}}$, in the dimuon frame

- $\phi$, between the two decay planes


formed by the $\mathrm{K} \pi$ and dimuon systems, in the $\mathrm{B}_{\mathrm{d}}$ frame.

- Measure:

$$
\begin{aligned}
\frac{1}{d \Gamma / d q^{2}} \frac{d^{4} \Gamma}{d \cos \theta_{L} d \cos \theta_{K} d \phi d q^{2}}= & \frac{9}{32 \pi}\left[F_{L} \cos ^{2} \theta_{K}+\frac{3\left(1-F_{L}\right)}{4} \sin ^{2} \theta_{K}+\frac{1-F_{L}}{4} \sin ^{2} \theta_{K} \cos 2 \theta_{L}\right. \\
& -F_{L} \cos ^{2} \theta_{K} \cos 2 \theta_{L}+S_{3} \sin ^{2} \theta_{K} \sin ^{2} \theta_{L} \cos 2 \phi \\
& +S_{4} \sin 2 \theta_{K} \sin 2 \theta_{L} \cos \phi+S_{5} \sin 2 \theta_{K} \sin \theta_{L} \cos \phi \\
& +S_{6} \sin ^{2} \theta_{K} \cos \theta_{L}+S_{7} \sin 2 \theta_{K} \sin \theta_{L} \sin \phi \\
& \left.+S_{8} \sin 2 \theta_{K} \sin 2 \theta_{L} \sin \phi+S_{9} \sin ^{2} \theta_{K} \sin ^{2} \theta_{L} \sin 2 \phi\right]
\end{aligned}
$$

The familiar forward-backward asymmetry is given by $A_{F B}=3 \mathrm{~S}_{6} / 4$. 
- The method to reduce hadronic form factor dependence is this: the $S_{i}$ depend on the form factors and have significant uncertainty at LO. Transform the $S_{i}$ using ratios constructed to cancel the form factor dependence at $L O$ :

$$
\begin{aligned}
& P_{1}=\frac{2 S_{3}}{1-F_{L}} \\
& P_{2}=\frac{2}{3} \frac{A_{F B}}{1-F_{L}} \\
& P_{3}=-\frac{S_{9}}{1-F_{L}} \\
& P^{\prime}{ }_{i=4,5,6,8}=\frac{S_{j=4,5,7,8}}{\sqrt{F_{L}\left(1-F_{L}\right)}}
\end{aligned}
$$

- All these parameters depend on the invariant mass squared of the dilepton system $\left(\mathrm{q}^{2}\right)$, so analyze data in 6 partially-overlapping $\mathrm{q}^{2}$ bins. 


\section{Analysis details:}

- Integrated luminosity $=20.3 \mathrm{fb}^{-1}$

- Trigger: 1,2 , or 3 muons

- Reconstruct muons: $\mathrm{p}_{\mathrm{T}}>3.5 \mathrm{GeV},|\eta|<2.5$

- Require: $\mu^{+} \mu^{-}$reconstruct to a common vertex

- Candidate kaon, pion tracks: $\mathrm{p}_{\mathrm{T}}>0.5 \mathrm{GeV}$ [no dedicated particle ID in ATLAS]

- Select $\mathrm{K}^{*}$ mesons: $\mathrm{p}_{\mathrm{T}}\left(\mathrm{K}^{*}\right)>3.0 \mathrm{GeV}, \mathrm{m}(\mathrm{K} \pi)$ within $[846,946] \mathrm{MeV}$.

- Reconstruct B candidate:

- Flavor assigned from $\mathrm{K}$ charge.

- Vertex $\mathrm{K}^{*}$ with $\mu^{+} \mu^{-}$

- Require consistent vectors: vector from PV to $B_{d}$ decay vertex, and $B_{d}$ momentum vector

- Suppress combinatorial bkg with lifetime significance cut: $\tau\left(\mathrm{B}_{\mathrm{d}}\right) / \sigma_{\tau}>12.5$

- Suppress partially recon decays with tight lower cut around nominal $\mathrm{B}_{\mathrm{d}}$ mass: $5150<\mathrm{m}(\mathrm{K} \pi \mu \mu)<5700 \mathrm{MeV}$

- to eliminate extra candidates per event, choose best match to $\mathrm{m}\left(\mathrm{K}^{*}\right)_{\mathrm{PDG}}$, and best $\mathrm{B}$ vertex fit.

- $\mathrm{q}^{2}$ bin range: $[0.04,6.0]$ excluding $[9.8,1.1]$ (to remove $\phi$ resonance)

- Compare data to a model using maximum likelihood, for Gaussian signal, with parameters taken from a control region, and $4 \mathrm{bkg}$ components

- To overcome low statistics, a "folding procedure" of transformations is used that exploits trigonometric relations among the angular parameters. 


\section{The models:}

- Ciuchini et al. (CFFMPSV) ${ }^{1}-$ QCD factorization framework to perform consistency checks of the LHCb data with theory expectations

- Descotes-Genon et al. (DHMV) ${ }^{2}-$ QCD factorization

- Jäger and Camalich (JC) $)^{3}$ QCD factorization, focus on impact of long distance corrections using a helicity amplitude approach

\section{Results:}

Good agreement except in $3 \mathrm{q}^{2}$ bins: $\mathrm{P}_{4}{ }_{4}$ and $\mathrm{P}_{5}{ }_{5}$ in $\mathrm{q}^{2}$ bin $[4.0,6.0]$ and $\mathrm{P}_{8}^{c}$ in $\mathrm{q}^{2}$ bin $[2.0,4.0]$.

The $\mathrm{P}_{4}{ }_{4}\left(\mathrm{P}_{5}\right)$ deviations are consistent with the LHCb observation and are $2.5(2.7)$ sigma from the DHMV model. All measurements are within 3 sigma of the SM theory band. They are also compatible with the $\mathrm{LHCb}$ result.

${ }^{1}$ JHEP 06 (2016)116, arXiv: 1512.07157 [hep-ph]

2 JHEP 12 (2014) 125, arXiv: 1407.8526 [hep-ph]

${ }^{3}$ JHEP 05 (2013) 043, arXiv: 1212.2263 [hep-ph]; PRD 93 (2016) 014028, arXiv: 1412.3183 [hep-pk] 
The 3 noted deviations, for ATLAS and $\mathrm{LHCb}$ data and theoretical models
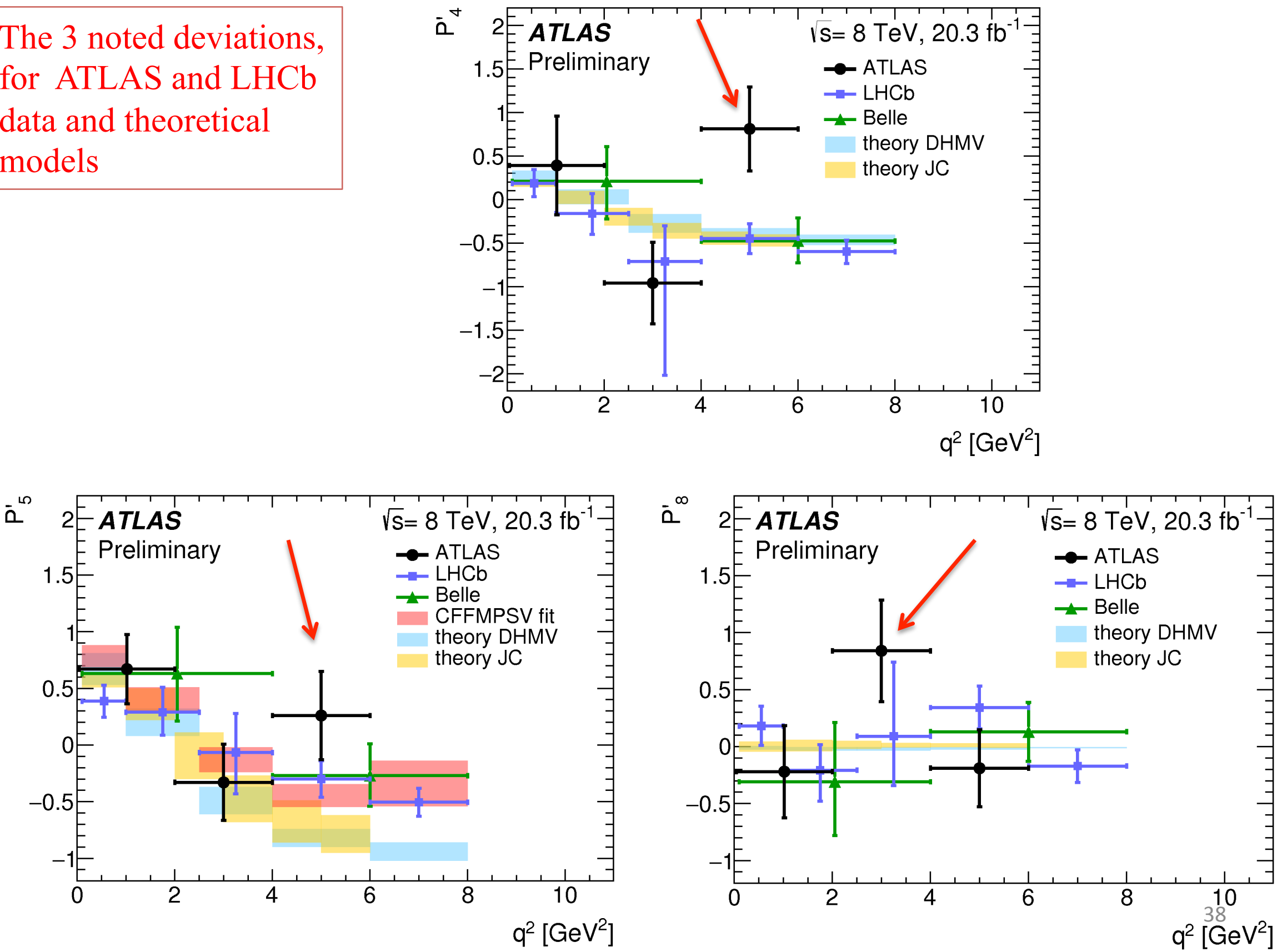
ATLAS presents 4 measurements using data recorded at ${ }_{s}=8 \mathrm{TeV}$ at the LHC. All are compared to contemporary models.

- Differential cross sections for b-hadron pair production - to improve the theoretical description of quarkonium production and to facilitate background subtractions in new physics searches.

- Prompt J/ $/ \psi$ pair production differential cross sections - to characterize double parton scattering as a probe of the gluon distribution in the proton, and to investigate correlations in the non-perturbative regime.

- Differential production cross sections for $\psi(2 S)$ and $X(3872)$, both observed in decays to $J / \psi \pi^{+} \pi^{-}$- a study of production mechanisms through examination of prompt and non-prompt signals.

- An angular analysis of $B_{d}^{0} \rightarrow K^{*} \mu^{+} \mu^{-}$decays - a potential probe of new physics contributions through penguin diagrams. 